\title{
Modeling the Impact of a Coronavirus Vaccine Among Low and High Risk Populations in the United States
}

\author{
Rachel Ruffin, Ariana Richardson, and Enahoro A. Iboi*
}

April 2021

\begin{abstract}
The novel coronavirus (COVID-19) was first reported in the U.S. on December 29, 2019 and has spread rapidly throughout the country, affecting individuals with varying severity due to their risk status. According to the Centers for Disease Control and Prevention, it is estimated that $45.4 \%$ of US adults are at higher risk for complications from coronavirus disease because of cardiovascular disease, diabetes, respiratory disease, hypertension, or cancer. In this study, we developed a mathematical model to assess the impact of a COVID-19 vaccine among low and high risk groups. Numerical simulations shows vaccinating both low and high risk groups simultaneously, rather than prioritizing the vaccine on high risk group only, further reduces the daily mortality. The result supports the need for an aggressive vaccination program, regardless of whether individuals are within the low or high risk population.
\end{abstract}

Keywords: COVID-19; non-pharmaceutical interventions; vaccinations; vaccine doses; pre-existing condition; high risk; low risk.

\section{Introduction}

The novel coronavirus (COVID-19) originated in Wuhan, China, where the first cases were recorded on December 29, 2019 [1]. Coronavirus failed to be contained and spread across the world, quickly becoming declared a pandemic. As of April 21, 2020, over 144 million people worldwide have been infected with COVID-19 and over 3 million people have died. Within the United States alone, almost 32 million people have contracted coronavirus and over 569,000 Americans have died [2]. A study conducted by Chen et al.[3] determined risk factors for COVID-19 death, using 1859 infected subjects. Findings showed that older age put participants at a higher risk for death, with 70 years old being the median age of the deceased. Also, $74 \%$ of the subjects who died from COVID-19 were male. Additionally, many of the deceased had pre-existing health conditions, with $50 \%$ of those who died having hypertension, compared to $29 \%$ of the surviving population having hypertension. In the same regard, $28 \%$ of those who died had diabetes, compared to $12 \%$ of the living participants having diabetes. Out of the deceased population, $8 \%$ had cancer, whereas only $3 \%$ of those who survived had cancer. The study also cited smoking history as a risk factor for COVID-19 death [3]. Additionally, the CDC cited that 45.4\% of U.S. adults are at a higher risk for COVID-19 complications due to cardiovascular disease, diabetes, respiratory disease, hypertension, or cancer [4].

There are currently three vaccines being used to stop the spread of COVID-19 in the United States: Pfizer-BioNTech, Moderna, and Johnson \& Johnson. Due to the limited supply of the vaccine to the general public, high risk groups and frontline essential workers have been prioritized for vaccination. These phase 1 groups include healthcare workers, long-term care facility staff and residents, education and childcare workers, military personnel, those with pre-existing health conditions, and adults 65 and older. Low risk individuals are classified as those who are nonessential workers, under the age of 65 and without pre-existing health conditions. As the vaccines become more available and most high risk group have been fully vaccinated, vaccination will continue for the other groups (low risk). Over 125 million Americans have received at least one dose of the coronavirus vaccine and almost 78.5 million individuals are fully vaccinated. Still, this only accounts for $37.9 \%$ and $23.6 \%$ of the total population, respectively [5]. Thus, an analysis into the population-level impact of COVID-19 vaccine administration among low and high risk populations is necessary considering the prioritization of a specific group (high risk), the large amount of unvaccinated individuals in the United States, vaccine supply challenges, and the global impact of the virus.

Previous research has been conducted using mathematical modeling to analyze the impact of a potential anti-COVID-19 vaccine on coronavirus transmission, such as in the model developed by Iboi et al. [6]. This study assumed a vaccine efficacy

*Corresponding author: Email: enahoroiboi@spelman.edu 
of $80 \%$ and concluded that the United States could rid itself of COVID-19 if at least $90 \%$ of the U.S. population is vaccinated and if non-pharmaceutical methods are also used in combination [6]. These studies, however, do not consider separating the population into low risk and high risk categories. In this work, we use mathematical modeling to assess the population-level impact of a COVID-19 vaccine of $95 \%$ efficacy among both high and low risk groups. This vaccine efficacy was determined following announcements from Pfizer and BioNTech on November 18, 2020 [7]. The parameters used in this model are estimated using available COVID-19 data for the United States from Johns Hopkins. The model is formulated in Section 2. The results are presented in Section 3 which include parameters estimation of the model, and numerical simulations of the model. Discussions and concluding remarks are presented in Section 4.

\section{Methods and Materials}

\subsection{Model Formulation}

For this study, we utilize a mathematical model to assess the impact of a COVID-19 vaccine among low and high risk populations in the United States. At time $t$, the total population $(N(t))$ can be divided into disjoint categories of low risk, unvaccinated susceptible $\left(S_{u}^{L}(t)\right)$, high risk, unvaccinated susceptible $\left(S_{u}^{H}(t)\right)$, low risk, vaccinated susceptible $\left(S_{v}^{L}(t)\right)$, high risk, vaccinated susceptible $\left(S_{v}^{H}(t)\right)$, low risk exposed $\left(E^{L}(t)\right)$, high risk exposed $\left(E^{H}(t)\right)$, low risk asymptomatic-Infectious $\left(A^{L}(t)\right)$, high risk asymptomatic-Infectious $\left(A^{H}(t)\right)$, low risk symptomatic-Infectious $\left(I^{L}(t)\right)$, high risk symptomatic-Infectious $\left(I^{H}(t)\right)$, low risk hospitalized $\left(J^{L}(t)\right)$, high risk hospitalized $\left(J^{H}(t)\right)$, and recovered $(R(t))$ individuals. Thus,

$N(t)=S_{u}^{L}(t)+S_{u}^{H}(t)+S_{v}^{L}(t)+S_{v}^{H}(t)+E^{L}(t)+E^{H}(t)+A^{L}(t)+A^{H}(t)+I^{L}(t)+I^{H}(t)+J^{L}(t)+J^{H}(t)+R(t)$.

The model is composed of the following nonlinear differential equations (where a dot represents differentiation with respect to time $t$ ):

$$
\begin{aligned}
\dot{S}_{u}^{L} & =-\left(\lambda_{L}+\lambda_{H}\right) S_{u}^{L}-\left(\omega+\psi_{1}\right) S_{u}^{L} \\
\dot{S}_{u}^{H} & =-\left(\lambda_{L}+\lambda_{H}\right) S_{u}^{H}+\omega S_{u}^{L}-\psi_{2} S_{u}^{H} \\
\dot{S}_{v}^{L} & =\psi_{1} S_{u}^{L}-\left(1-\epsilon_{v}\right)\left(\lambda_{L}+\lambda_{H}\right) S_{v}^{L}-\omega S_{v}^{L} \\
\dot{S}_{v}^{H} & =\psi_{2} S_{u}^{H}-\left(1-\epsilon_{v}\right)\left(\lambda_{L}+\lambda_{H}\right) S_{v}^{H}+\omega S_{v}^{L} \\
\dot{E}^{L} & =\left(\lambda_{L}+\lambda_{H}\right)\left[S_{u}^{L}+\left(1-\epsilon_{v}\right) S_{v}^{L}\right]-\left(\sigma_{L}+\omega\right) E^{L} \\
\dot{E}^{H} & =\left(\lambda_{L}+\lambda_{H}\right)\left[S_{u}^{H}+\left(1-\epsilon_{v}\right) S_{v}^{H}\right]+\omega E^{L}-\sigma_{H} E^{H} \\
\dot{A}^{L} & =r \sigma_{L} E^{L}-\left(\gamma_{a}^{L}+\omega\right) A^{L} \\
\dot{A}^{H} & =g \sigma_{H} E^{H}+\omega A^{L}-\gamma_{a}^{H} A^{H} \\
\dot{I}^{L} & =(1-r) \sigma_{L} E^{L}-\left(\omega+\phi_{I}^{L}+\gamma_{I}^{L}+\delta_{I}^{L}\right) I^{L} \\
\dot{I}^{H} & =(1-g) \sigma_{H} E^{H}+\omega I^{L}-\left(\phi_{I}^{H}+\gamma_{I}^{H}+\delta_{I}^{H}\right) I^{H} \\
\dot{J}^{L} & =\phi_{I}^{L} I^{L}-\left(\gamma_{j}^{L}+\delta_{L}\right) J^{L} \\
\dot{J}^{H} & =\phi_{I}^{H} I^{H}-\left(\gamma_{j}^{H}+\delta_{H}\right) J^{H} \\
\dot{R} & =\gamma_{a}^{L} A^{L}+\gamma_{a}^{H} A^{H}+\gamma_{I}^{L} I^{L}+\gamma_{I}^{H} I^{H}+\gamma_{j}^{L} J^{L}+\gamma_{j}^{H} J^{H}
\end{aligned}
$$

where the forces of infection are given by

$$
\lambda_{L}=\beta_{L}\left(\frac{I^{L}+\eta_{L} A^{L}}{N}\right), \lambda_{H}=\beta_{H}\left(\frac{I^{H}+\eta_{H} A^{H}}{N}\right) .
$$

\section{Data Collection and Analysis}

The model (2.1) uses estimates for some of the non vaccine related parameters obtained from the literature (as indicated in Table 3). Fitting the model (2.1) provided other parameter values in the context of a COVID-19 vaccine and the observed cumulative data for the United States. We obtained from the John Hopkins University COVID-19 database, the cumulative 


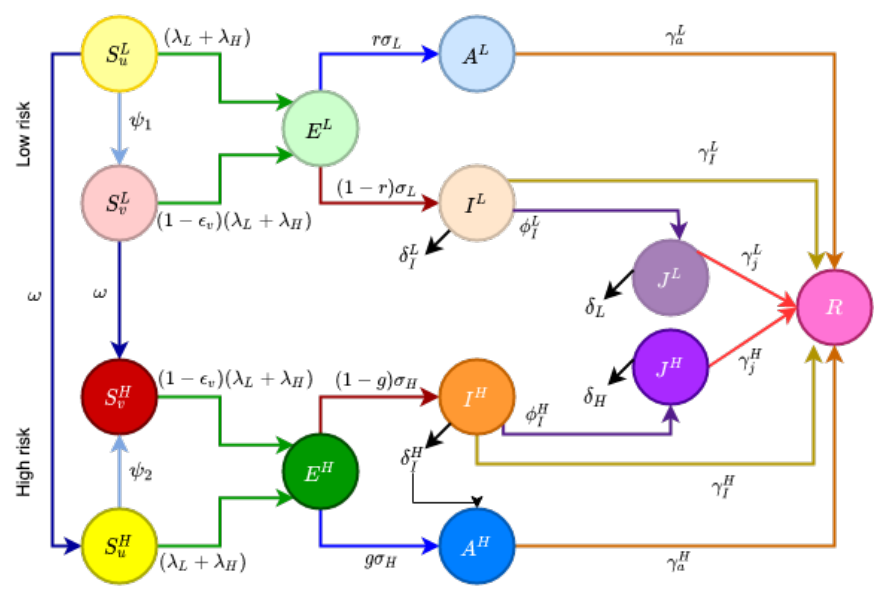

Figure 1: Flowchart of COVID-19 Vaccination Model

mortality data from January 12, 2020 to February 24, 2021 [2]. We fit the model for this time period of the pandemic in order to correctly capture the trends observed in the cumulative mortality data to examine vaccination impact in low and high risk communities. Our decision to fit the model to mortality data, rather than case data, is due to the evidence of underreporting and under-testing of COVID-19 cases in various countries such as France, Italy, United States, Iran, and Spain [8, 9]. Therefore, the mortality data may provide a better indicator for COVID-19 case spread. The process of data-fitting involves applying the standard nonlinear least squares approach using the fmincon Optimization Toolbox embedded in MATLAB. The estimated values of the unknown parameters are tabulated in Table 3. Figure 2 depicts the fitting of the observed and predicted cumulative mortality for the United States.

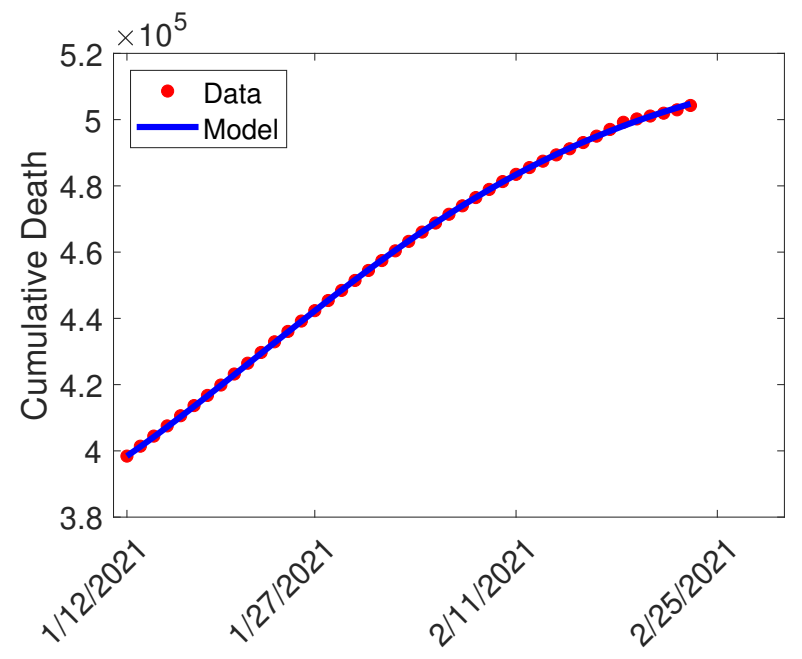

Figure 2: Data fitting of the model (2.1) using the cumulative mortality data for the United States from January 12, 2021 to February 24, 2021.

\section{Results}

The population-level impact of the COVID-19 vaccine on the cumulative and daily mortality will now be explored through simulations of model 2.1 using parameter values in Table 3 unless otherwise stated. It is worth mentioning that the PfizerBioNTech and Moderna vaccines with approximately 95\% efficacy were administered to high risk individuals in the early stages of vaccination, before Johnson \& Johnson vaccination began. Our study assumed a daily vaccination of 3 million people [10]. Figure 3a shows the impact of a 5, 10, and 15-fold increase in vaccination rate from the baseline value on the cumulative mortality. Our simulation results project 498,163 cumulative mortality with a 5-fold increase in vaccination rate (which corresponds to fully vaccinating approximately 15 million people per day) from the baseline value by February 

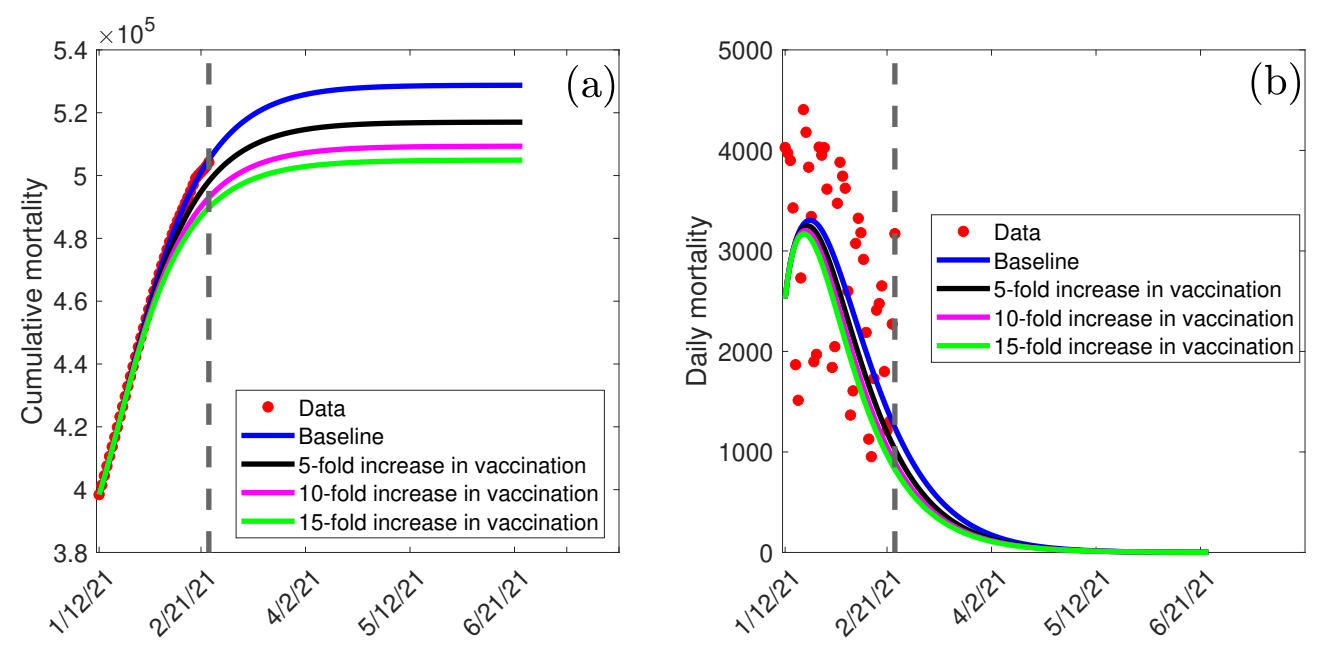

Figure 3: Model simulation displaying cumulative and daily mortality in the U.S. when vaccination rates are increased by 5 , 10, and 15 fold. Dashed vertical line depict outcomes for 120 days after February 24, 2021.
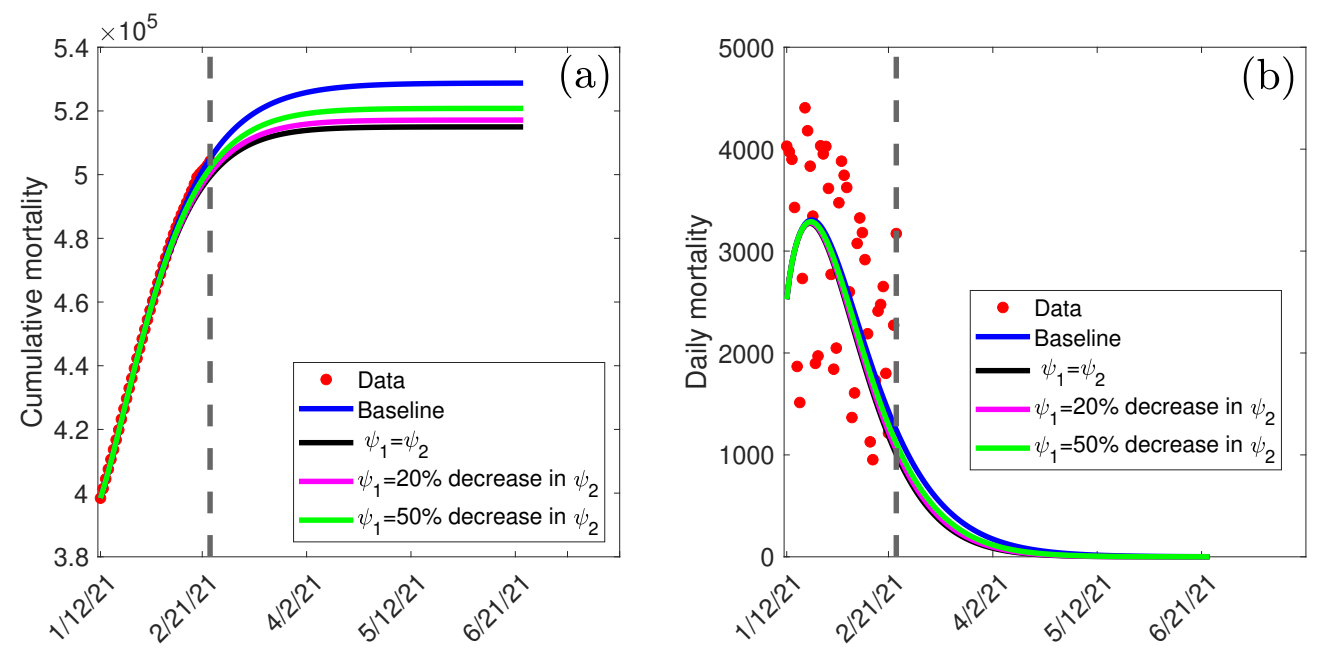

Figure 4: Model simulation displaying cumulative and daily mortality in the U.S. when low and high risk vaccination rates are altered. Dashed vertical line depict outcomes for 120 days after February 24, 2021. 
24,2021 . This is a $1.36 \%$ decrease in cumulative mortality when compared to the baseline scenario $(505,031)$. A similar trend was observed with a 10 -fold increase in vaccination rate (which corresponds to vaccinating about 30 million people daily) from the baseline mortality value shows a projected 492,978 cumulative mortality by February 24, 2021. This is a $2.39 \%$ decrease in cumulative mortality when compared to the baseline scenario. Further, a 15-fold increase in vaccination rate (which corresponds to vaccinating almost 45 million people daily) from the baseline value shows a projected 489,706 cumulative mortality from the baseline scenario, which is a $3.0 \%$ decrease from the baseline figure. Figure $3 \mathrm{~b}$ shows the impact of a 5, 10, and 15-fold increase in vaccination rate from the baseline value of 1,244 on the daily mortality. Our simulation results project 1,027 daily mortality with a 5-fold increase in vaccination rate from the baseline value by February 24,2021 . This is a $17.5 \%$ decrease in daily mortality when compared to the baseline scenario. A similar trend was observed with a 10-fold increase in vaccination rate from the baseline mortality value shows a projected 896 daily mortality by February 24,2021 . This is a $27.9 \%$ decrease in daily mortality when compared to the baseline scenario. Further, a 15 -fold increase in vaccination rate from the baseline value shows a projected 828 daily mortality, which is a $33.4 \%$ decrease from the baseline figure.

Figure 4a depicts the impact of vaccinating both low and high risk groups on the cumulative mortality. Here we assume approximately 3 million daily vaccinations of high risk group. A 20\% decrease in high risk vaccination rate which corresponds to vaccinating 2.4 million low risk group daily, and a $50 \%$ decrease in high risk vaccination rate which corresponds to vaccinating 1.5 million low risk group daily. Our simulation results project 502,041 cumulative mortality (1,113 daily mortality) from the baseline value by February 24, 2021 when 3 million high risk individuals and 1.5 million low risk individuals are vaccinated daily. This is a $0.6 \%$ decrease in cumulative mortality (10.5\% daily) when compared to the baseline scenario. A similar trend was observed when 3 million high risk individuals and 2.4 million low risk individuals are vaccinated daily. Our result shows a projected 500,409 cumulative mortality (1,046 daily mortality) from the baseline mortality value by February 24,2021 . This is a $0.9 \%$ decrease in cumulative mortality (15.9\% daily) when compared to the baseline scenario. Further, when low risk vaccination rate is equal that of the high risk group (which corresponds to vaccinating 3 million high risk individuals and 3 million low risk individuals daily) from the baseline value shows a projected 499,380 cumulative mortality ( 1,005 daily mortality) from the baseline scenario. This is a $1.1 \%$ decrease in cumulative mortality $(19.2 \%)$. Figure $4 \mathrm{~b}$ shows the need to consider vaccinating both risk groups at the beginning of the vaccination program rather than prioritizing the vaccine on high risk group only. The result from Figure 3 and 4 supports the need for an aggressive vaccination program, regardless of whether individuals are within the low or high risk population.

\section{Summary and Conclusions}

To gain a stronger understanding of the impact of the COVID-19 vaccine among low and high risk populations, we developed a mathematical model to assess the vaccine effect on cumulative and daily mortality rates in the United States. Our simulations portrayed the need for an aggressive vaccination in both low and high risk communities in order to reduce COVID-19 induced mortality. For example, a 15-fold increase in vaccination rate (which corresponds to vaccinating almost 45 million people daily) from the baseline value shows a projected 489,706 cumulative mortality by February 24, 2021, which is a 3.0\% decrease in the cumulative mortality from the baseline scenario. We assessed the impact of vaccinating both low and high risk groups simultaneously on the cumulative mortality. Our results show that vaccinating the low risk population at the rate of only $50 \%$ of the high risk population (corresponding to 3 million high risk individuals and 1.5 million low risk individuals daily) results in a $0.6 \%$ decrease in cumulative mortality.

In conclusion, this study shows the need to complement the fight to eliminate COVID-19 in the United States through vaccination in addition to other non-pharmaceutical interventions, such as face mask usage and social distance. Vaccinating individuals, regardless of their risk status, will further decrease the both the cumulative and daily mortality in the United States. The increase of vaccination rates of high and low risk populations should be a priority in order to avoid an ongoing increase in daily cases and deaths being recorded.

\section{Acknowledgements}

RR, AR, and EAI would like to acknowledge and thank our partners of The Boeing Company and the Thurgood Marshall College Fund, for their charitable, capacity grant in support of the Math RaMP Program at Spelman College, via the Boeing | TMCF HBCU Investment. We would also like to acknowledge the MAA Tensor Women and Mathematics Program for support of the Math RaMP Program at Spelman College. EAI would like to acknowledge the support of the National Science Foundation (Award \#1761945). 


\section{Authors contributions}

RR, AR, and EAI conceived the study; RR, AR, and EAI designed the model; AR and RR collected and analysed the data; RR and EAI performed the numerical simulations; RR, AR, and EAI drafted the manuscript. All authors read and approved the final manuscript. Ethics approval was not required as all data used in the manuscript are publicly available.

\section{Conflicts of Interest}

All authors declare no conflicts of interest.

Table 1: Description of the state variables of the model (2.1)

\begin{tabular}{|l|l|}
\hline State Variable & Description (L = low risk, $\mathrm{H}=$ high risk) \\
\hline$S_{u}^{L}$ & Population of low risk, unvaccinated susceptible individuals \\
$S_{u}^{H}$ & Population of high risk, unvaccinated susceptible individuals \\
$S_{v}^{L}$ & Population of low risk, vaccinated susceptible individuals \\
$S_{v}^{H}$ & Population of high risk, vaccinated susceptible individuals \\
$E^{L}$ & Population of low risk, exposed individuals \\
$E^{H}$ & Population of high risk, exposed individuals \\
$A^{L}$ & Population of low risk, asymptomatic-Infectious individuals \\
$A^{H}$ & Population of high risk, asymptomatic-Infectious individuals \\
$I^{L}$ & Population of low risk, symptomatic-Infectious individuals \\
$I^{H}$ & Population of high risk, symptomatic-Infectious individuals \\
$H$ & Population of hospitalized individuals \\
$J^{L}$ & Population of low risk hospitalized individuals \\
$J^{H}$ & Population of high risk hospitalized individuals \\
$R$ & Population of recovered individuals \\
\hline
\end{tabular}

Table 2: Description of the parameters of the model (2.1)

\begin{tabular}{|l|l|}
\hline Parameter & Description \\
\hline$\delta_{I}^{L}, \delta_{j}^{L}\left(\delta_{I}^{H}\right)\left(\delta_{j}^{H}\right)$ & Mortality rate, symptomatic low risk (high risk) \\
$\phi$ & Hospitalization rate \\
$\gamma_{a}\left(\gamma_{I}\right)\left(\gamma_{H}\right)$ & Recovery rate, Asymptomatic (symptomatic/hospitalized) \\
$\omega$ & Rate of transition from low risk to high risk unvaccinated (vaccinated) population \\
$\sigma$ & Rate of transition from exposed to infectious \\
$r$ & Low risk recovery rate \\
$g$ & High risk recovery rate \\
$\psi$ & Vaccination rate \\
$\epsilon_{v}$ & Vaccine efficacy \\
$\beta_{L}\left(\beta_{H}\right)$ & Transmission probability of low risk (high risk) population \\
\hline
\end{tabular}


Table 3: Parameter values for the model (2.1)

\begin{tabular}{|l|l|l|}
\hline Parameter & Value & Reference \\
\hline $\mathrm{r} / \mathrm{g}$ & 0.65 & {$[6]$} \\
\hline$\psi_{1}$ & 0.039 & {$[5]$} \\
\hline$\psi_{2}$ & 0 & {$[5]$} \\
\hline$\epsilon_{v}$ & $0.95(0.94)(0.72)$ & {$[11]$} \\
\hline$\sigma^{H}$ & 0.19 & {$[12]$} \\
\hline$\sigma^{L}$ & 0.2 & {$[12]$} \\
\hline$\gamma_{I}^{L}$ & 0.15 & {$[4,12]$} \\
\hline$\gamma_{I}^{H}$ & 0.14 & {$[4,12]$} \\
\hline$\gamma_{A}^{L}$ & 0.15 & {$[4,12]$} \\
\hline$\gamma_{A}^{H}$ & 0.14 & {$[4,12]$} \\
\hline$\gamma_{J}$ & $1 / 14$ & {$[12]$} \\
\hline$\eta_{L}$ & 0.25 & {$[12]$} \\
\hline$\eta_{H}$ & 0.25 & {$[12,13]$} \\
\hline$\beta_{L}$ & 0.7906 & Fitted \\
\hline$\beta_{H}$ & 0.2562 & Fitted \\
\hline$\omega$ & 0.0385 & Fitted \\
\hline$\phi_{I}^{L}$ & 0.083 & {$[12]$} \\
\hline$\phi_{I}^{H}$ & 0.1245 & Estimated \\
\hline$\delta_{I}^{L}$ & 0.02052 & {$[12]$} \\
\hline$\delta_{I}^{H}$ & 0.026676 & Estimated \\
\hline$\delta_{j}^{L}$ & 0.015 & {$[12]$} \\
\hline$\delta_{j}^{H}$ & 0.0195 & Estimated \\
\hline
\end{tabular}

\section{References}

[1] Q. Li, X. Guan, P. Wu, X. Wang, L. Zhou, Y. Tong, R. Ren, K. S. Leung, E. H. Lau, J. Y. Wong, et al., "Early transmission dynamics in wuhan, china, of novel coronavirus-infected pneumonia," New England Journal of Medicine (2020).

[2] J. H. U. . Medicine, "Coronavirus resource center," https: //coronavirus.jhu.edu/map.html (2020 (accessed November 19, 2020)).

[3] L. Chen, J. Yu, W. He, L. Chen, G. Yuan, F. Dong, W. Chen, Y. Cao, J. Yang, L. Cai, et al., "Risk factors for death in 1859 subjects with covid-19," Leukemia 34, 2173-2183 (2020).

[4] M. L. Adams, D. L. Katz, and J. Grandpre, "Population-based estimates of chronic conditions affecting risk for complications from coronavirus disease, united states," Emerging infectious diseases 26, 1831 (2020).

[5] C. for Disease Control and Prevention, "Covid-19 vaccinations in the united states," https://covid.cdc.gov/ covid-data-tracker/\#vaccinations (2021 (accessed April 15, 2021)).

[6] A. B. Gumel, E. A. Iboi, and C. N. Ngonghala, "Will an imperfect vaccine curtail the covid-19 pandemic in the us?" medRxiv (2020).

[7] Pfizer and BioNTech, "Pfizer and biontech conclude phase 3 study of covid-19 vaccine candidate, meeting all primary efficacy endpoints," https://www.pfizer.com/news/press-release/press-release-detail/ pfizer-and-biontech-conclude-phase-3-study-covid-19-vaccine (2020 (accessed November 19, 2020)).

[8] M. S. Lau, B. Grenfell, M. Thomas, M. Bryan, K. Nelson, and B. Lopman, "Characterizing superspreading events and age-specific infectiousness of sars-cov-2 transmission in georgia, usa," Proceedings of the National Academy of Sciences 117, 22430-22435 (2020).

[9] C. N. Ngonghala, E. A. Iboi, and A. B. Gumel, "Could masks curtail the post-lockdown resurgence of covid-19 in the US?” Mathematical biosciences 329, 108452 (2020). 
[10] "U.s. covid vaccinations slow as more states pass half of residents jabbed," (2020). Online Version

[11] C. for Disease Control and Prevention, "Different vaccines," https://www.cdc.gov/coronavirus/ 2019-ncov/vaccines/different-vaccines.html (2021 (accessed April 15, 2021)).

[12] S. E. Eikenberry, M. Mancuso, E. Iboi, T. Phan, K. Eikenberry, Y. Kuang, E. Kostelich, and A. B. Gumel, "To mask or not to mask: Modeling the potential for face mask use by the general public to curtail the covid-19 pandemic," Infectious Disease Modelling (2020).

[13] M. A. Johansson, T. M. Quandelacy, S. Kada, P. V. Prasad, M. Steele, J. T. Brooks, R. B. Slayton, M. Biggerstaff, and J. C. Butler, "Sars-cov-2 transmission from people without covid-19 symptoms," JAMA network open 4, e2035057e2035057 (2021). 